\begin{tabular}{|c|l|}
\hline Title & Impurity levels and resonant transmission of acoustic phonons in a double barrier system \\
\hline Author(s) & Mizuno, Seiji; Tamura, Shin-ichiro \\
\hline Citation & $\begin{array}{l}\text { Physical Review B, 45(23), 13423 13430 } \\
\text { https://doi.org/10.1103/PhysRevB.45.13423 }\end{array}$ \\
\hline Issue Date & 1992-06-15 \\
\hline Doc URL & http://hdl.handle.net/2115/5709 \\
\hline Rights & Copyright $\odot 1992$ A merican Physical Society \\
\hline Type & article \\
\hline File Information & PRB45-23.pdf \\
\hline
\end{tabular}

Instructions for use 


\title{
Impurity levels and resonant transmission of acoustic phonons in a double-barrier system
}

\author{
Seiji Mizuno and Shin-ichiro Tamura \\ Department of Engineering Science, Hokkaido University, Sapporo 060, Japan
}

(Received 11 February 1992)

\begin{abstract}
We study theoretically the resonant transmission of acoustic phonons in a double-barrier system for phonons consisting of a bulk material sandwiched between periodic superlattices. The phonon transmission rate and the resonance condition in this system are derived analytically based on the transfer-matrix method. We show that the phonons in a frequency gap of the superlattices can be transmitted through the whole system without attenuation, if the frequency of incident phonons satisfies the resonance condition. Based on the Green's-function method, we also show that the resonant frequencies coincide with the impurity levels associated with the lattice vibrations localized near the embedded bulk layer.
\end{abstract}

\section{INTRODUCTION}

The phonon-dispersion relation in a periodic superlattice is obtained by folding the dispersion curves for a bulk material back into the mini-Brillouin-zone determined by the periodicity of the superlattice. ${ }^{1}$ In the folded dispersion relation, frequency gaps are generally formed at the center and the boundary of the mini-Brillouin-zone. We can control the size of the mini-Brillouin-zone by changing the length of the unit period of the superlattice. This implies that we can also set the frequency gaps in an experimentally accessible frequency range ( $\lesssim 1 \mathrm{THz}$ ) much lower than those of bulk solids.

So far, the propagation of acoustic phonons in superlattices with various stacking order of constituent layers has been studied both experimentally and theoretically. ${ }^{2}$ An interesting feature studied recently is the resonant transmission of phonons in $A B A$ multisuperlattice structures composed of two kinds of periodic superlattices $A$ and $B .{ }^{3}$ In the present paper, we study the phonon transmission in a system where the $B$ superlattice is replaced by a bulk material. An advantage of this simpler system is that we can develop analytical calculations for the resonance condition and elucidate its physical origin.

Thus, the system that we consider consists of a bulk material sandwiched between the same periodic superlattices. A schematic frequency-band diagram for this system is shown in Fig. 1. In this figure, the hatched regions indicate the frequency gaps of superlattices. The phonons within these frequency gaps cannot be transmitted through the system. In other words, the superlattices act as barriers for phonons with frequencies between $\omega_{L}$ and $\omega_{H}$. Thus, we can regard the system shown in Fig. 1 as a double-barrier system for phonons in the forbidden gap. This structure is similar to the double-barrier quantumwell structure for electrons. ${ }^{4}$ This similarity suggests the possibility for designing various phonon optics devices such as a phonon mirror, phonon resonator, and phonon filter suggested by Narayanamurti several years ago. ${ }^{5}$ In the present paper, from the viewpoint of realizing such devices, we study theoretically the transmission charac- teristics of acoustic phonons in this double-barrier system.

The outline of this paper is as follows. In Sec. II, we first introduce a transfer matrix to formulate mathematically the dynamics of phonons in a multilayered system. Next, we present an analytical expression for the phonon transmission rate by the transfer-matrix method. Then, the resonance phenomena of phonons in the doublebarrier system are predicted both analytically and numerically. In Sec. III, to analyze the physical origin of the resonant transmission of phonons through barriers, we consider a corresponding system in which the width of both barriers is infinity. In this case, the system we consider is a single-well structure and the equation of motion for the lattice displacement at interfaces becomes formally the same as the Schrödinger equation for the onedimensional tight-binding model with two neighboring impurities. We solve the latter equation by using the Green's-function method and obtain the equations giving the frequencies of the impurity levels. These equations are found to be identical to the conditions for the resonant transmission derived in Sec. II. We also discuss the

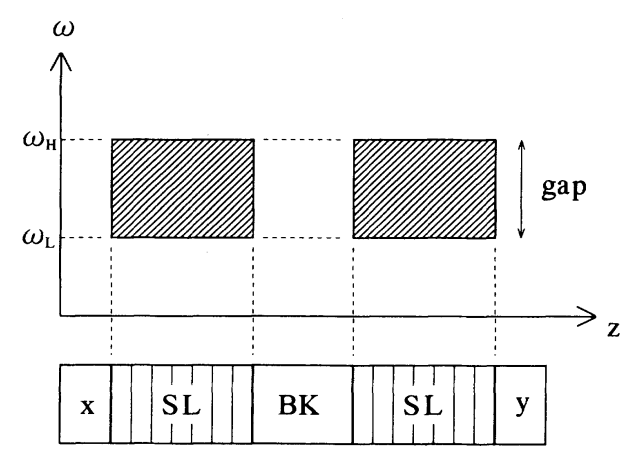

FIG. 1. Schematic frequency band along a direction $z$, perpendicular to the layer interfaces of the double-barrier system. The hatched regions indicate the frequency gaps. The parts SL, $\mathrm{BK}, x$, and $y$ mean the periodic superlattice, bulk material, substrate, and phonon detector, respectively. 
number of the impurity levels in a given structure graphically. In Sec. IV, a summary and conclusions are given.

\section{TRANSMISSION \\ IN THE DOUBLE-BARRIER SYSTEM}

In this section, we study the transmission of acoustic phonons in a double-barrier system. In particular, we consider the case where the wave vector $\mathbf{k}$ of the phonons is perpendicular to the layer interfaces of the system. In this case, three phonon modes are decoupled from one another if the interfaces are a mirror-symmetry plane. For simplicity, we consider this case and treat only one mode of phonons, e.g., the longitudinal mode. Furthermore, we adopt the continuum model for the lattice vibrations. This model is valid for sub-THz phonons because acoustic branches in the phonon-dispersion relation for most of the semiconductor superlattices are liner in $k$ $(=|\mathbf{k}|)$ in the frequency range below $1 \mathrm{THz}$.

In Sec. II A, we present the general expression for the phonon transmission rate in the double-barrier structure by calculating the products of transfer matrices analytically. In Sec. II B, we present a numerical example for the phonon transmission based on our formula.

\section{A. Analytical expressions for the transfer matrix and transmission rate}

A schematic picture of the double-barrier system is shown in Fig. 2. The structure grown on a substrate $x$ and having a detector layer on top of it is divided into three parts, which are called SL, BK, and SL. Two SL parts have the same structure consisting of alternate stacking of semiconductors $A$ and $B$, while the BK part consists of the bulk semiconductor $A$. The thicknesses of

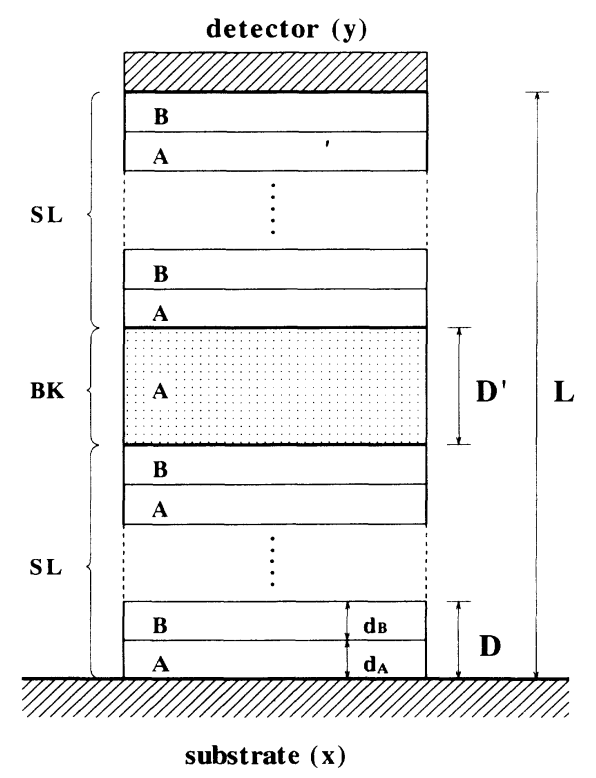

FIG. 2. Schematic picture of the double-barrier system. This system is divided into three parts: SL, BK, and SL. Two SL parts have the same structure consisting of alternate stacking of semiconductors $A$ and $B$, while the BK part consists of the bulk semiconductor $A$. the $A$ and $B$ layers in the SL parts are denoted by $d_{A}$ and $d_{B}$, respectively, and $D\left(=d_{A}+d_{B}\right)$ is the unit period of the SL part. The thicknesses of the BK part and the whole system are denoted by $D^{\prime}$ and $L$, respectively.

In the continuum model, the lattice displacement $U_{i}(z)$ and stress $S_{i}(z)$ for the acoustic mode are expressed in terms of linear combinations of the transmitted and reflected waves:

$$
\begin{aligned}
& U_{i}(z)=c_{i}^{t} e^{i k_{i} z}+c_{i}^{r} e^{-i k_{i} z}, \\
& S_{i}(z)=i \omega Z_{i}\left(c_{i}^{t} e^{i k_{i} z}-c_{i}^{r} e^{-i k_{i} z}\right) .
\end{aligned}
$$

Here, $i$ is an index specifying constituent layers; $c_{i}^{t}$ and $c_{i}^{r}$ are the amplitudes of the transmitted and reflected waves, respectively; $k_{i}$ is the wave number; $Z_{i}=\rho_{i} v_{i}$ is the acoustic impedance given by the product of the mass density $\rho_{i}$ and the sound velocity $v_{i} ; \omega=k_{i} v_{i}$ is the frequency.

The lattice displacement $U_{i}(z)$ and stress $S_{i}(z)$ should be continuous at each interface of adjacent layers. These boundary conditions can be expressed in terms of the transfer matrix and lead to the relations among the displacement $U_{D}(L)$ and stress $S_{D}(L)$ at the detector-SL interface, and $U_{S}(0)$ and $S_{S}(0)$ at the substrate-SL interface;

$$
\left[\begin{array}{l}
U_{D}(L) \\
S_{D}(L)
\end{array}\right]=T_{W}\left(\begin{array}{l}
U_{S}(0) \\
S_{S}(0)
\end{array}\right),
$$

where the transfer matrix $T_{W}$ for the whole system can be written as a product of the transfer matrices for three parts SL, BK, and SL,

$$
T_{W} \equiv T_{\mathrm{SL}}(N) T_{\mathrm{BK}} T_{\mathrm{SL}}(N) \text {, }
$$

with $N$ indicating the number of periodicity of the SL parts. The transfer matrix $T_{\mathrm{BK}}$ for the $\mathrm{BK}$ part is given by

$$
T_{\mathrm{BK}}=\left(\begin{array}{cc}
\cos \gamma & \frac{1}{\omega Z_{A}} \sin \gamma \\
-\omega Z_{A} \sin \gamma & \cos \gamma
\end{array}\right),
$$

with $\gamma=k_{A} D^{\prime}$. The transfer matrix $T_{\mathrm{SL}}$ for the SL part with $N$ periods is expressed as

$$
T_{\mathrm{SL}}(N)=\left(T_{B} T_{A}\right)^{N},
$$

where the transfer matrices $T_{A}$ and $T_{B}$ for the constituent layers $A$ and $B$ have the similar form as $T_{\mathrm{BK}}$,

$$
\begin{aligned}
& T_{A}=\left(\begin{array}{cc}
\cos \alpha & \frac{1}{\omega Z_{A}} \sin \alpha \\
-\omega Z_{A} \sin \alpha & \cos \alpha
\end{array}\right], \\
& T_{B}=\left(\begin{array}{cc}
\cos \beta & \frac{1}{\omega Z_{B}} \sin \beta \\
-\omega Z_{B} \sin \beta & \cos \beta
\end{array}\right],
\end{aligned}
$$

with $\alpha=k_{A} d_{A}$ and $\beta=k_{B} d_{B}$. Thus, we have 


$$
T_{B} T_{A}=\left(\begin{array}{cc}
\lambda & \frac{1}{\omega Z_{A}} \sigma \\
\omega Z_{A} \zeta & \mu
\end{array}\right),
$$

where

$$
\begin{aligned}
& \lambda=\cos \alpha \cos \beta-\frac{Z_{A}}{Z_{B}} \sin \alpha \sin \beta, \\
& \sigma=\sin \alpha \cos \beta+\frac{Z_{A}}{Z_{B}} \cos \alpha \sin \beta, \\
& \zeta=-\sin \alpha \cos \beta-\frac{Z_{B}}{Z_{A}} \cos \alpha \sin \beta, \\
& \mu=\cos \alpha \cos \beta-\frac{Z_{B}}{Z_{A}} \sin \alpha \sin \beta .
\end{aligned}
$$

The analytical expression for $\left(T_{B} T_{A}\right)^{N}$ is presented in Ref. 3; the result is

$$
T_{\mathrm{SL}}(N)=\left(\begin{array}{cc}
\frac{\lambda-\mu}{2} S(N)+C(N) & \frac{1}{\omega Z_{A}} \sigma S(N) \\
\omega Z_{A} \zeta S(N) & -\frac{\lambda-\mu}{2} S(N)+C(N)
\end{array}\right),
$$

where

$$
\begin{aligned}
& S(N)= \begin{cases}\frac{\sin (N \theta)}{\sin \theta} & \text { for }\left|\frac{\mu+\lambda}{2}\right| \leq 1 \\
\frac{\sinh (N \theta)}{\sinh \theta} & \text { for } \frac{\mu+\lambda}{2}>1 \\
(-1)^{N+1} \frac{\sinh (N \theta)}{\sinh \theta} & \text { for } \frac{\mu+\lambda}{2}<-1,\end{cases} \\
& C(N)= \begin{cases}\cos (N \theta) & \text { for } \quad \frac{\mu+\lambda}{2} \mid \leq 1 \\
\cosh (N \theta) & \text { for } \frac{\mu+\lambda}{2}>1 \\
(-1)^{N} \cosh (N \theta) & \text { for } \frac{\mu+\lambda}{2}<-1 .\end{cases}
\end{aligned}
$$

Here, $\theta$ is defined by

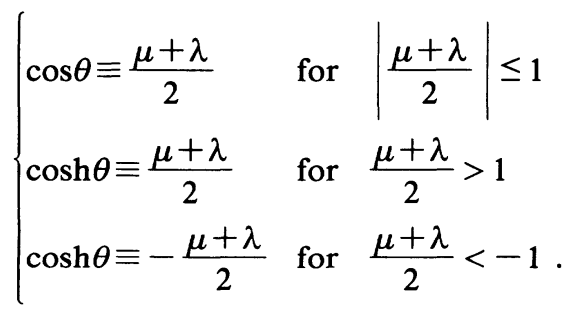

The frequencies satisfying the condition $|(\mu+\lambda) / 2|$ $>1[|(\mu+\lambda) / 2| \leq 1]$ are inside the frequency gaps (bands). By inserting Eqs. (5) and (14) into Eq. (4), we can obtain the transfer matrix $T_{W}$ for the whole system. The calculation of $T_{W}$ is straightforward but the explicit ex- pression is lengthy. Thus, we write down here only the expression of $T_{W}$ for $|(\mu+\lambda) / 2|>1$ because this is the case of our main concern:

$$
T_{W}=\left(\begin{array}{cc}
a & \frac{1}{\omega Z_{A}} b \\
\omega Z_{A} c & d
\end{array}\right),
$$

where

$$
\begin{aligned}
a= & \frac{1}{2}\left[1 \pm \frac{\lambda-\mu}{2 \sinh \theta}\right] G_{ \pm} e^{2 N \theta}+\frac{1}{2}\left(1 \mp \frac{\lambda-\mu}{2 \sinh \theta}\right] G_{\mp} e^{-2 N \theta} \\
& -\frac{(\lambda-\mu)(\xi-\sigma)}{4 \sinh ^{2} \theta} \sin \gamma \\
b= & \pm \frac{\sigma}{2 \sinh \theta}\left(G_{ \pm} e^{2 N \theta}-G_{\mp} e^{-2 N \theta}\right) \\
& +\left(1-\frac{\sigma(\zeta-\sigma)}{2 \sinh ^{2} \theta}\right) \sin \gamma \\
c= & \pm \frac{\zeta}{2 \sinh \theta}\left(G_{ \pm} e^{2 N \theta}-G_{\mp} e^{-2 N \theta}\right) \\
& -\left[1+\frac{\zeta(\zeta-\sigma)}{2 \sinh ^{2} \theta}\right) \sin \gamma \\
d= & \frac{1}{2}\left[1 \mp \frac{\lambda-\mu}{2 \sinh ^{2} \theta}\right] G_{ \pm} e^{2 N \theta}+\frac{1}{2}\left(1 \pm \frac{\lambda-\mu}{2 \sinh \theta}\right] G_{\mp} e^{-2 N \theta} \\
& +\frac{(\lambda-\mu)(\zeta-\sigma)}{4 \sinh ^{2} \theta} \sin \gamma \\
G_{ \pm} & =\cos \gamma \pm \frac{\zeta-\sigma}{2 \sinh \theta} \sin \gamma
\end{aligned}
$$

The upper sign corresponds to the case $(\mu+\lambda) / 2>1$ and the lower sign to $(\mu+\lambda) / 2<-1$. In terms of $a, b, c$, and $d$, the transmission rate $t$ of phonons through the whole system is expressed as

$$
t=\frac{4 \frac{Z_{y}}{Z_{x}}}{\left(\frac{Z_{y}}{Z_{A}} b-\frac{Z_{A}}{Z_{x}} c\right)^{2}+\left(d+\frac{Z_{y}}{Z_{x}} a\right)^{2}} .
$$

The details of the derivation of the above equation is presented in Ref. 3.

\section{B. A numerical example}

As a numerical example, we choose (100) AlAs/GaAs superlattices for the SL parts and AlAs for the BK part. The unit period of the SL parts consists of $(6 \mathrm{ML}$ AlAs)/(6 ML GaAs), and the number of period is $N=12$. The thickness of the BK part is the same as the SL part (i.e., $D^{\prime}=N D$ ). The other parameters are as follows: the thickness of one monolayer (ML) in the SL part is $2.83 \AA$ in the [100] direction for both AlAs $(=A)$ and GaAs $(=B)$ (i.e., $d_{A}=d_{B}=2.83 \AA$ ); the mass density and longitudinal sound velocity are $5.36 \mathrm{~g} / \mathrm{cm}^{3}$ and $4.71 \mathrm{~km} / \mathrm{s}$ for GaAs, and $3.76 \mathrm{~g} / \mathrm{cm}^{3}$ and $5.65 \mathrm{~km} / \mathrm{s}$ for AlAs (i.e., $\rho_{A}=3.76 \mathrm{~g} / \mathrm{cm},{ }^{3} \rho_{B}=5.36 \mathrm{~g} / \mathrm{cm},{ }^{3} v_{A}=5.65 \mathrm{~km} / \mathrm{s}$, 


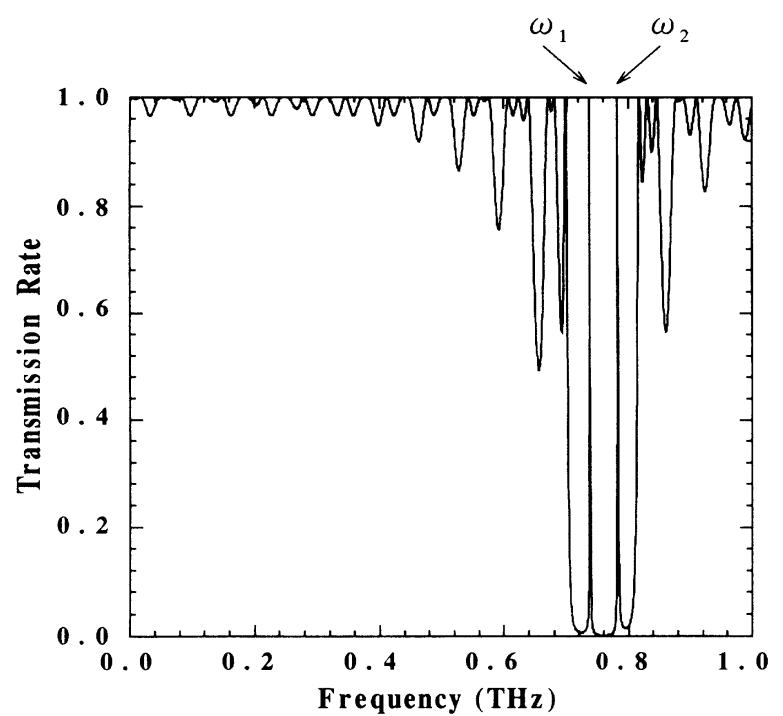

FIG. 3. Frequency dependence of the longitudinal phonon transmission rate in a double-barrier system. The parameters used are in the text.

$\left.v_{B}=4.71 \mathrm{~km} / \mathrm{s}\right)$.

In Fig. 3, we show the frequency dependence of the longitudinal phonon transmission rate through the whole system calculated from Eq. (24). We find a large dip $(0.72-0.80 \mathrm{THz})$ in the given frequency range. This dip is due to the Bragg reflection of the phonons in the SL part. The frequency at the center of this dip predicted by the first-order Bragg condition $v=\frac{1}{2}\left(d_{A} / v_{A}+d_{B} / v_{B}\right)^{-1}$ is $0.76 \mathrm{THz}$. This value is in good agreement with the frequency at the center of the dip in Fig. 3.

In addition to the appearance of this dip, the most noticeable feature in transmission is the existence of two sharp enhancements within the dip. To see the details of this structure, the transmission rate in the dip is enlarged in Fig. 4. These enhancements in transmission correspond to the resonance characteristic of the system we study.

Here, we discuss the origin of these resonant transmissions quantitatively based on the analytical expression for the transmission rate derived in Sec. II A. Because our interest is in the phonons in the gap of the SL part, we may calculate the transmission rate for $|(\mu+\lambda) / 2|>1$. The relevant transfer matrix in this case is given in Eqs. (18)-(23). Inserting Eqs. (18)-(23) into Eq. (24), we can obtain the exact expression for $T_{W}$ under the condition $|(\mu+\lambda) / 2|>1$. The result is

$$
t=\frac{4}{P^{2}+Q^{2}}
$$

where

$$
\begin{aligned}
P= & G_{ \pm} e^{2 N \theta}+G_{\mp} e^{-2 N \theta}, \\
Q= & \pm \frac{\widetilde{\sigma}-\widetilde{\xi}}{2 \sinh \theta}\left\{G_{ \pm} e^{2 N \theta}-G_{\mp} e^{-2 N \theta}\right\} \\
& +\left[\frac{Z_{x}}{Z_{A}}+\frac{Z_{A}}{Z_{x}}-\frac{\widetilde{\sigma}-\xi}{2 \sinh ^{2} \theta}(\xi-\sigma)\right] \sin \gamma,
\end{aligned}
$$

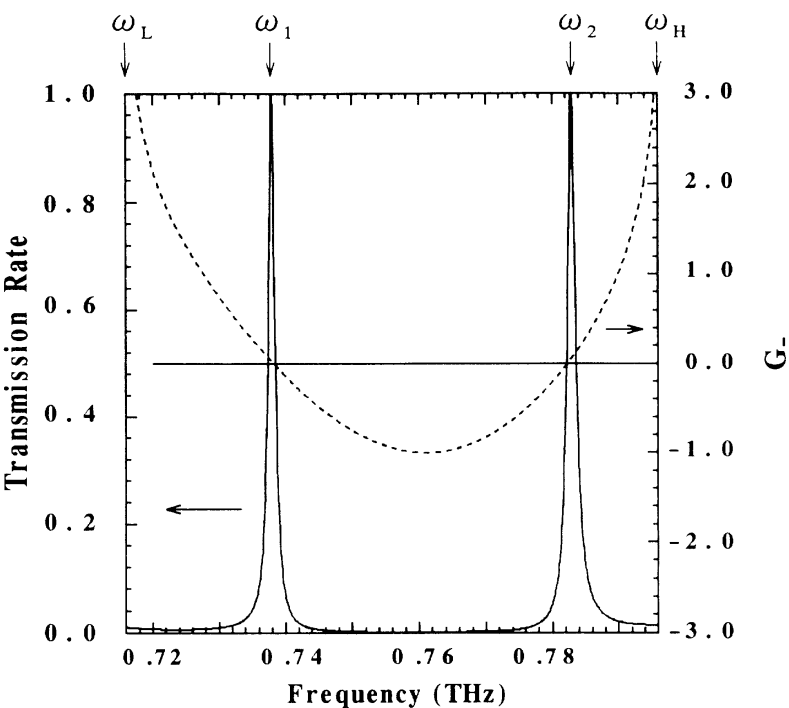

FIG. 4. The longitudinal phonon transmission rate (solid line) and the function $G_{-}$(dotted line) within the lowest frequency gap in the double-barrier system.

with $\widetilde{\sigma}=\left(Z_{x} / Z_{A}\right) \sigma$ and $\widetilde{\xi}=\left(Z_{A} / Z_{x}\right) \xi$. Here, we have assumed for simplicity that the substrate and detector are the same semiconductors, i.e., $Z_{x}=Z_{y}$. Hence, as the number of periods $N$ of the SL parts becomes larger, both $P$ and $Q$ increase exponentially, or the transmission rate becomes zero in proportion with $e^{-4 N \theta}$. This is valid as far as the coefficients $G_{ \pm}$of $e^{2 N \theta}$ in Eqs. (26) and (27) are nonzero. However, if these coefficients are zero, that is,

$$
G_{+}=0 \text { for } \frac{\mu+\lambda}{2}>1
$$

or

$$
G_{-}=0 \text { for } \frac{\mu+\lambda}{2}<-1
$$

is satisfied, the transmission rate has a finite value,

$t=4\left[\left[\frac{Z_{x}}{Z_{A}}+\frac{Z_{A}}{Z_{x}}-\frac{\widetilde{\sigma}-\widetilde{\xi}}{2 \sinh ^{2} \theta}(\xi-\sigma)\right] \sin \gamma\right]^{-2}$,

for a large $N$. Therefore, Eqs. (28) and (29) should be known as the resonance condition. The physical origin of the resonance will be clarified in the next section.

In Fig. 4, we show the frequency dependence of $G_{-}$ (relevant to the frequency range shown in Fig. 3). As expected, we can find a good coincidence of the frequencies at the sharp enhancements in transmission with the fre-

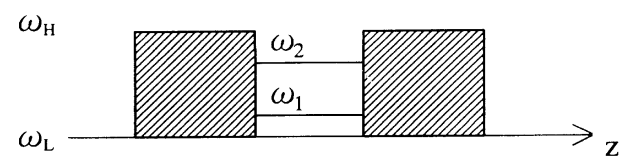

FIG. 5. Discrete levels induced by the sandwiched bulk materials. 


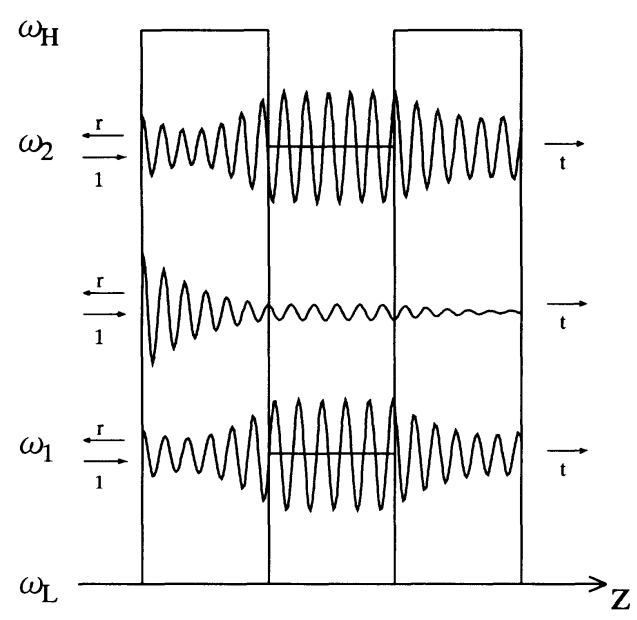

FIG. 6. The profiles of the real part of the amplitude of the phonons within the gap.

quencies satisfying $G_{-}=0$. The very small deviation between frequency satisfying $G_{-}=0$ and the location of the transmission peak is due to the finite number of periodicity $(N=12)$ of the SL part assumed, i.e., the terms of $e^{-2 N \theta}$ in Eqs. (26) and (27) have small but still seizable contributions. If $N$ becomes larger, the deviation becomes much smaller.

Our results show that even phonons inside the frequency gap of the SL part can be transmitted through the whole system when they satisfy the resonance condition (28) or (29). A schematic picture for this result is shown in Fig. 5. The hatched regions indicate the frequency gaps for the SL parts. The frequencies $\omega_{1}$ and $\omega_{2}$ stand for the resonance frequencies at which the sharp enhancements in transmission occur. The diagram of Fig. 5 is very similar to the double-barrier quantum-well structure for electrons in which the resonant tunneling occurs.

The profiles of the real part of the amplitude of the phonons within the gap $\left(\omega_{L}<\omega<\omega_{H}\right)$ are shown in Fig. 6; these profiles correspond to $\omega=\omega_{1}, \omega_{1}<\omega<\omega_{2}$, and $\omega=\omega_{2}$. In the case $\omega \neq \omega_{1}, \omega_{2}$, the real part of the amplitude decays as the phonons propagate through both of the SL parts, implying that the phonons cannot propagate through the system if the barriers are appropriately thick. However, for $\omega=\omega_{1}, \omega_{2}$, the profiles of the amplitude have quite different shapes from those at $\omega \neq \omega_{1}, \omega_{2}$, and the phonons can propagate through both barriers without attenuation. This behavior is characteristic of the resonant transmission.

\section{IMPURITY LEVELS OF PHONONS}

In Sec. II B we considered the phonon transmission in the double-barrier system. We can regard the BK part as an impurity embedded in the perfect periodic superlattice. In this picture, it is expected that the resonance frequencies given by the solutions of Eqs. (28) and (29) agree with the frequencies of impurity levels of phonons generated by the BK part. Based on the Green's-function method, ${ }^{6}$ we show that this is indeed the case.

In this section, we consider the case where the number of the period $N$ of the SL parts becomes infinity, i.e., the widths of both barriers are infinity. As discussed in Sec. II A, the continuity conditions for the lattice displacement and stress can be expressed as

$$
\left[\begin{array}{l}
U_{n+1} \\
S_{n+1}
\end{array}\right]=\left[\begin{array}{cc}
\lambda_{n} & \frac{1}{\omega Z_{A}} \sigma_{n} \\
\omega Z_{A} \xi_{n} & \mu_{n}
\end{array}\right]\left[\begin{array}{l}
U_{n} \\
S_{n}
\end{array}\right] .
$$

Here, $U_{n}$ and $S_{n}$ are the displacement and stress at the $n$th interface, respectively; $\lambda_{n}, \sigma_{n}, \zeta_{n}$, and $\mu_{n}$ are the elements of the transfer matrix associated with the segment of the system located between the $n$th and the $(n+1)$ th interfaces. When the relevant segment is the BK part, the transfer matrix is given by Eq. (5), i.e.,

$$
\lambda_{l}=\mu_{l}=\cos \gamma, \quad \sigma_{l}=\sin \gamma, \quad \zeta_{l}=-\sin \gamma,
$$

where we assume that the BK part is located between the $l$ th and the $(l+1)$ th interfaces. On the other hand, when this segment is a unit period of the SL part, the corresponding transfer matrix is given by Eq. (9), i.e., for $n \neq 1$,

$$
\lambda_{n}=\lambda, \mu_{n}=\mu, \sigma_{n}=\sigma, \zeta_{n}=\zeta .
$$

From Eq. (31), we have

$$
\begin{aligned}
& U_{n+1}=\lambda_{n} U_{n}+\frac{\sigma_{n}}{\omega Z_{A}} S_{n}, \\
& U_{n}=\lambda_{n-1} U_{n-1}+\frac{\sigma_{n-1}}{\omega Z_{A}} S_{n-1}, \\
& S_{n}=\omega Z_{A} \zeta_{n-1} U_{n-1}+\mu_{n-1} S_{n-1} .
\end{aligned}
$$

Eliminating $S$ from these equations, we obtain the equation governing the lattice displacement, ${ }^{7}$

$$
\frac{1}{\sigma_{n}} U_{n+1}+\frac{1}{\sigma_{n-1}} U_{n-1}=\left(\frac{\lambda_{n}}{\sigma_{n}}+\frac{\mu_{n-1}}{\sigma_{n-1}}\right) U_{n} .
$$

Here, we introduce the following parameters:

$$
\begin{aligned}
& E=\mu+\lambda, \quad V=\frac{\sigma}{\sin \gamma}-1, \\
& \varepsilon_{1}=\lambda-\sigma \cot \gamma, \quad \varepsilon_{2}=\mu-\sigma \cot \gamma .
\end{aligned}
$$

With this notation, Eq. (35) can be rewritten as

$$
\begin{aligned}
& U_{l-1}+\varepsilon_{1} U_{l}+(1+V) U_{l+1}=E U_{l}, \\
& (1+V) U_{l}+\varepsilon_{2} U_{l+1}+U_{l+2}=E U_{l+1}, \\
& U_{n-1}+U_{n+1}=E U_{n} \quad(n \neq l, l+1) .
\end{aligned}
$$

These equations are mathematically equivalent to the Schrödinger equation for the one-dimensional tightbinding model with neighboring two impurities. For this model, the Hamiltonian can be written as

$$
H=H_{0}+H_{1},
$$

where $H_{0}$ is the periodic tight-binding Hamiltonian with the site energy 0 and unit transfer integral, 


$$
H_{0}=\sum_{n}\{|n+1\rangle\langle n|+| n\rangle\langle n+1|\},
$$

and $H_{1}$ is the perturbation arising from the two neighboring impurities,

$$
\begin{aligned}
H_{1}= & \varepsilon_{1}|l\rangle\left\langle l\left|+\varepsilon_{2}\right| l+1\right\rangle\langle l+1| \\
& +V\{|l\rangle\langle l+1|+| l+1\rangle\langle l|\} .
\end{aligned}
$$

Here, each state $|n\rangle$ is an atomiclike orbital centered at the site $n$, or originally the amplitude of the phonons at the $n$th interface; $\varepsilon_{1}$ and $\varepsilon_{2}$ are the site energy of the $l$ th and $(l+1)$ th impurity sites, respectively, and $V$ is the transfer integral between the two neighboring impurity sites. The eigenvalues and eigenfunctions of the periodic tight-binding Hamiltonian $H_{0}$ have the well-known form

$$
\begin{aligned}
& \varepsilon_{q}=2 \cos (q D), \\
& \mid q)=\frac{1}{\sqrt{N}} \sum_{n} e^{i q n D}|n\rangle,
\end{aligned}
$$

where $q$ is the wave number restricted in the first miniBrillouin-zone.

Our purpose is to obtain the discrete levels induced by the two impurities. The most convenient way to find these discrete levels is to find the poles of the Green's function $G$ corresponding to $H$. The Green's function is defined by

$$
G(E) \equiv(E-H)^{-1}=\left(E-H_{0}-H_{1}\right)^{-1} .
$$

Similarly, we define the Green's function $G_{0}$ in the homogeneous system associated with $H_{0}$ :

$$
G_{0}(E) \equiv\left(E-H_{0}\right)^{-1} \text {. }
$$

We can expand $G$ in terms of $H_{1}$ and $G_{0}$ as

$$
\begin{aligned}
G & =\left[1-\left(E-H_{0}\right)^{-1} H_{1}\right]^{-1}\left(E-H_{0}\right)^{-1} \\
& =\left[1-G_{0} H_{1}\right]^{-1} G_{0} \\
& =G_{0}+G_{0} H_{1} G_{0}+G_{0} H_{1} G_{0} H_{1} G_{0}+\cdots
\end{aligned}
$$

or

$$
G=G_{0}+G_{0} T G_{0},
$$

where the $t$ matrix $T$ is defined by

$$
T \equiv H_{1}+H_{1} G_{0} H_{1}+H_{1} G_{0} H_{1} G_{0} H_{1}+\cdots \text {. }
$$

Taking account of the explicit expression of $H_{1}$ [Eq. (40)], we can write

$$
\begin{aligned}
T & =H_{1}+H_{1} \sum_{n}|n\rangle\left\langle n\left|G_{0} \sum_{m}\right| m\right\rangle\langle m| H_{1}+\cdots \\
& =H_{1}+H_{1}|\alpha\rangle\left\langle\alpha\left|G_{0}\right| \alpha\right\rangle\langle\alpha| H_{1}+\cdots
\end{aligned}
$$

where $|\alpha\rangle$ denotes the row vector $(|l\rangle,|l+1\rangle)$ and the matrices $\left\langle\alpha\left|G_{0}\right| \alpha\right\rangle$ and $\left\langle\alpha\left|H_{1}\right| \alpha\right\rangle$ are

$$
\begin{aligned}
& \left\langle\alpha\left|G_{0}\right| \alpha\right\rangle=\left(\begin{array}{ll}
\left\langle l\left|G_{0}\right| l\right\rangle & \left\langle l\left|G_{0}\right| l+1\right\rangle \\
\left\langle l+1\left|G_{0}\right| l\right\rangle & \left\langle l+1\left|G_{0}\right| l+1\right\rangle
\end{array}\right), \\
& \left\langle\alpha\left|H_{1}\right| \alpha\right\rangle=\left(\begin{array}{ll}
\varepsilon_{1} & V \\
V & \varepsilon_{2}
\end{array}\right) .
\end{aligned}
$$

Thus, we obtain from Eq. (48)

$$
\begin{aligned}
\langle\alpha|T| \alpha\rangle=\left\langle\alpha\left|H_{1}\right| \alpha\right\rangle+ & \left\langle\alpha\left|H_{1}\right| \alpha\right\rangle \\
& \times\left\langle\alpha\left|G_{0}\right| \alpha\right\rangle\left\langle\alpha\left|H_{1}\right| \alpha\right\rangle+\cdots \\
=\left\langle\alpha\left|H_{1}\right| \alpha\right\rangle\{1 & \left.-\left\langle\alpha\left|G_{0}\right| \alpha\right\rangle\left\langle\alpha\left|H_{1}\right| \alpha\right\rangle\right\}^{-1} .
\end{aligned}
$$

From Eqs. (46) and (51) we find that the poles of $G$ are given by

$$
\operatorname{det}\left\{1-\left\langle\alpha\left|G_{0}\right| \alpha\right\rangle\left\langle\alpha\left|H_{1}\right| \alpha\right\rangle\right\}=0 \text {. }
$$

The Green's function $G_{0}$ defined by Eq. (44) can be written in terms of the eigenfunction $\mid q$ ) of $H_{0}$ as

$$
G_{0}=\sum_{q} \frac{\mid q)(q \mid}{E-\varepsilon_{q}} .
$$

With the use of Eqs. (42) and (53), the matrix elements of $G_{0}$ are

$$
\begin{aligned}
\left\langle i\left|G_{0}\right| j\right\rangle & =\sum_{q} \frac{\langle i| q)(q|j\rangle}{E-\varepsilon_{q}} \\
& =\frac{D}{2 \pi} \int_{-\pi / D}^{\pi / D} \frac{e^{i q D(i-j)}}{E-2 \cos (q D)} d q .
\end{aligned}
$$

This integration can be carried out analytically and we have

$$
\begin{aligned}
\left\langle i\left|G_{0}\right| j\right\rangle & = \begin{cases}\frac{\left[E / 2-\sqrt{(E / 2)^{2}-1}\right]^{|i-j|}}{2 \sqrt{(E / 2)^{2}-1}} & \text { for } \frac{E}{2}>1 \\
-\frac{\left[E / 2+\sqrt{(E / 2)^{2}-1}\right]^{|i-j|}}{2 \sqrt{(E / 2)^{2}-1}} & \text { for } \frac{E}{2}<-1\end{cases} \\
& \equiv g_{|i-j|} .
\end{aligned}
$$

As in Eq. (17), we define $\theta$ by

$$
\cosh \theta=\left\{\begin{array}{ccc}
\frac{E}{2} & \text { for } & \frac{E}{2}>1 \\
-\frac{E}{2} & \text { for } & \frac{E}{2}<-1
\end{array}\right.
$$

[Eq. (56) is the same as Eq. (17), because $E$ is defined by $\mu+\lambda]$. Then, Eq. (55) is rewritten as

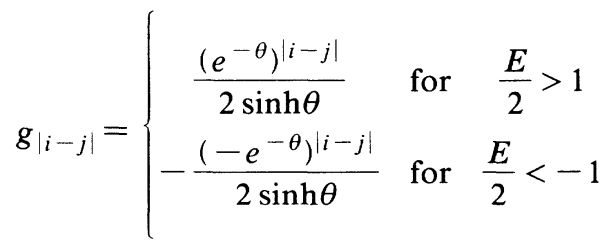

and the matrix $\left\langle\alpha\left|G_{0}\right| \alpha\right\rangle$ defined by Eq. (49) can be written as

$$
\left\langle\alpha\left|G_{0}\right| \alpha\right\rangle=\left(\begin{array}{ll}
g_{0} & g_{1} \\
g_{1} & g_{0}
\end{array}\right) .
$$

By inserting Eqs. (50) and (58) into Eq. (52), the equation which gives the poles of $G$ takes the form

$1-2 g_{1} V+\left(g_{1}^{2}-g_{0}^{2}\right)\left(V^{2}-\varepsilon_{1} \varepsilon_{2}\right)-\left(\varepsilon_{1}+\varepsilon_{2}\right) g_{0}=0$. 
Finally, substituting Eqs. (36) and (57) into Eq. (59), we obtain

$$
\cos \gamma \pm \frac{\zeta-\sigma}{2 \sinh \theta} \sin \gamma=0
$$

where the upper sign corresponds to the case where $(\mu+\lambda) / 2>1$ and the lower sign to $(\mu+\lambda) / 2<-1$. Equation (60) is identical to the resonance condition $G_{ \pm}=0$ [see Eqs. (23), (28), and (29)] given in Sec. II.

We also discuss the number of discrete levels given by the solutions of Eq. (60). Equation (60) is rewritten as

$$
-\cot \gamma= \pm \frac{\xi-\sigma}{2 \sinh \theta} \text {. }
$$

In Fig. 7, we show the frequency dependence of both sides of Eq. (61) within the frequency gap. The righthand side of Eq. (61) is a monotonically increasing or decreasing function of frequency ranging from $-\infty$ to $\infty$ $(\sinh \theta=0$ at the edges of the gap). The left-hand side is a monotonically increasing periodic function of $\gamma=k_{A} D^{\prime}$, whose period in inversely proportional to $D^{\prime}$. Hence, in each frequency gap there exists at least one intersection between these functions, or the solution of Eq. (61). In the present case, there exist two intersections which give the solutions of Eq. (61), i.e., the discrete frequencies $\omega_{1}$ and $\omega_{2}$. If the thickness $D^{\prime}$ of the BK part increases, the period of $-\cot \gamma$ decreases. Therefore, the number of the intersections increases. (If we double the thickness $D^{\prime}$, three intersections emerge, or there exist three impurity levels for $D^{\prime}=2 N D$.) On the other hand, if $D^{\prime}$ decreases, the number of the intersections decreases. This result is similar to the fact that the number of bound states of electrons in a single potential well structure increases with increasing well width.

Next, we calculate the amplitude of phonons at the frequencies corresponding to the discrete levels. Because these levels are obtained from the equation [see Eq. (45)]

$$
1-G_{0} H_{1}=0 \text {, }
$$

we can write for the $n$th state $\langle n|$,

$$
\langle n|\left(1-G_{0} H_{1}\right)=0 \text {. }
$$

This equation is rewritten as

$$
\begin{aligned}
\langle n| & =\langle n| G_{0} H_{1} \\
& =\left\langle n\left|G_{0}\right| \alpha\right\rangle\left\langle\alpha\left|H_{1}\right| \alpha\right\rangle\langle\alpha| \\
& =g_{n-l}\left(\varepsilon_{1}\langle l|+V\langle l+1|\right)+g_{n-l-1}\left(V\langle l|+\varepsilon_{2}\langle l+1|\right) .
\end{aligned}
$$

Therefore, the $n$th component $U_{n}=\langle n \mid b\rangle$ of the eigenfunction $(b)$ of $H$ is expressed as

$$
U_{n}=g_{n-l}\left(\varepsilon_{1} U_{l}+V U_{l+1}\right)+g_{n-l-1}\left(V U_{l}+\varepsilon_{2} U_{l+1}\right) .
$$

Green's function $g_{n}$ depends on the site index $n$ as

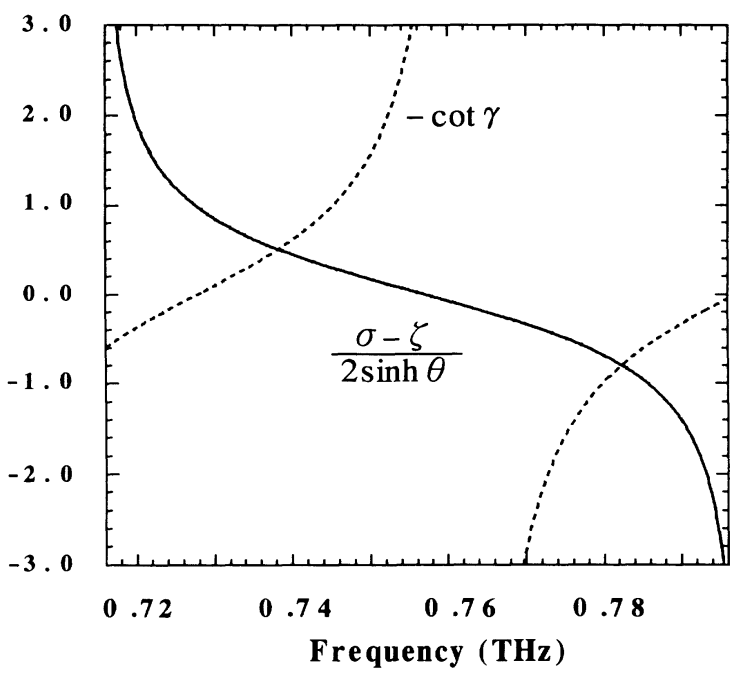

FIG. 7. Graphical solutions of Eq. (61).

$g_{n} \sim e^{-\ln \mid \theta}$ [see Eq. (57)]. Consequently, the amplitude at the interface of each layer decays exponentially on either side of the impurity site. That is, phonons at the impurity level exhibit a localized character.

\section{SUMMARY AND CONCLUDING REMARKS}

In the present work, we have derived analytical expressions for the transmission rate and the resonance condition in the double-barrier system for phonons. The results show that the phonons in the frequency gaps of the SL part are transmitted through both barriers without attenuation, when the frequency of the incident phonons coincides with that of discrete levels. These levels are caused by lattice vibrations localized at the sandwiched bulk material. We have also derived an equation giving the frequencies of these impurity levels.

These results signify that the double-barrier system we consider is analogous to the double-barrier system for electrons in which resonant tunneling occurs. This similarity suggests the potential for designing various phonon optics devices. In particular, the sharp resonant transmission in a wide frequency gap can be used to design a phonon resonator for the detection or generation of monochromatic high-frequency phonons. Our analytical expression for the transmission rate will be useful for this purpose.

\section{ACKNOWLEDGMENTS}

One of us (S.M.) was partly supported by a Research Aid of the Inoue Foundation for Science and had useful discussions with $\mathrm{K}$. Nakao and H. Hiramoto. Another (S.T.) was partly supported by the Suhara Memorial Foundation and by a Grant-in-Aid for Scientific Research from the Ministry of Education, Science, and Culture, Japan (Grant No. 03650001). 
${ }^{1}$ A. S. Barker, Jr., J. L. Merz, and A. C. Gossard, Phys. Rev. B 17, 3181 (1978).

${ }^{2}$ For a recent review, see S. Tamura, in Phonons 89 , edited by S. Hunklinger, W. Ludwig, and G. Weiss (World Scientific, Singapore, 1989), p. 703.

${ }^{3}$ S. Tamura, Phys. Rev. B 43, 12646 (1991); S. Mizuno and S. Tamura, ibid. 45, 734 (1992).
${ }^{4}$ L. L. Chang, L. Esaki, and R. Tsu, Appl. Phys. Lett. 24, 593 (1974). For a review, see F. Capasso, K. Mohammed, and A. Y. Cho, IEEE J. Quantum Electron. QE-22, 1853 (1986).

${ }^{5} \mathrm{~V}$. Narayanamurti, Science 213, 717 (1981).

${ }^{6}$ E. N. Economou, Green's Functions in Quantum Physics (Springer-Verlag, Berlin, 1983).

${ }^{7}$ S. Tamura, Phys. Rev. B 39, 1261 (1989). 